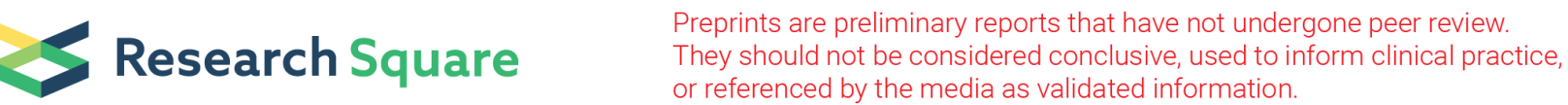

\section{Comparison of the effect of the graft with double strands peroneus longus tendon and with the four strands hamstring tendon on reconstruction of the posterior cruciate ligament injury}

Yue Lu

Affiliated Hospital of Nantong University

Hua Xu

Affiliated Hospital of Nantong University

Zhenyu Zhou

Affiliated Hospital of Nantong University

Lei Fang

Affiliated Hospital of Nantong University

Ran Tao ( $\sim$ tr2020sci@sina.com )

Affiliated Hospital of Nantong University

\section{Research}

Keywords: double-stranded long peroneal tendon, posterior cruciate ligament, four strands hamstring tendon, arthroscope

Posted Date: May 5th, 2020

DOI: https://doi.org/10.21203/rs.3.rs-26038/v1

License: (c) (i) This work is licensed under a Creative Commons Attribution 4.0 International License.

Read Full License 


\section{Abstract \\ Background}

This prospective study was to analyze the clinical effect of double-stranded long peroneal tendon and the four strands hamstring tendon in reconstruction of posterior cruciate ligament (PCL), and to investigate the effectiveness and safety of the two surgical approaches in reconstruction of PCL, so as to provide guidance for clinical treatment of PCL injury.

\section{Methods}

Total 48 patients with complete rupture of PCL treated in our hospital from July 2015 to February 2017 were selected and divided into the Group A (reconstructed with double strands peroneus longus tendon, 25 cases) and the Group B (four strands hamstring tendon, 23 cases) according to the different grafts.

\section{Results}

The intraoperative time for tendon extraction in the Group A (20.87 $\pm 2.07 \mathrm{~min})$ was significantly shorter than that in the Group B $(26.79 \pm 2.32 \mathrm{~min})$. Compared with pre-operation, the tibial posterior displacement of the two groups decreased significantly 24 months after operation, and IKDC score, Lysholm score and Tegner score increased significantly. Nevertheless, the IKDC score, Lysholm score, Tegner score, AOFAS score and Maryland score between the two groups before and after operation were no significant differences. Compared with the quadrupled hamstring tendon, the double-stranded peroneal longus tendon has the advantages of simple and safe tendon extraction.

\section{Conclusions}

Both surgical methods are effective for the treatment of PCL injury. When the four strands hamstring tendon is accidental (tendon rupture or tendon variation) or ineffective, the peroneal longus tendon will be a good choice.

\section{Introduction}

Posterior cruciate ligament (PCL), as the strongest ligament in the knee joint, is the most important joint structure to maintain the stability of the knee joint and occupies an important proportion in knee joint injury [1]. PCL injury will lead to the destruction of the knee joint's backward stability and rotation stability, seriously affecting the physiological function of the affected lower limbs, and greatly reducing the quality of life of patients [2]. If the PCL is not repaired in time after injury, it will further lead to structural damage of the medial and lateral meniscus, knee cartilage and other knee joints. 
At present, the treatment of PCL injury mainly includes conservative treatment and reconstruction treatment [3]. In patients who need reconstruction therapy, how to select appropriate materials has been one of the hot topics [4]. In addition to resembling the PCL structure, the reconstructed material must be able to replace the physiological function of PCL and restore the stability of knee joint to the maximum extent [5]. The main materials for PCL reconstruction are allogenic tendons, artificial ligaments and autogenous tendons [6]. Allogenic tendons have not been widely used in clinic due to the risk of rejection and transmission of diseases [7]. Artificial ligament is abrasive and debris is easy to cause synovitis of knee joint with a short service life. Buy its long-term effect has not been verified clinically and its clinical application is also limited [8].

Autogenous tendon has become one of the most widely used transplantation materials because of its similar structure and no immune rejection [9]. Four strands Hamstring tendon is favored by many scholars because of its good biological similarity and has been widely used in clinic [10]. Nevertheless, there is a potential risk of destroying the medial structure of the knee joint, and reports of donor saphenous nerve injuries have occasionally emerged. Recently, some studies have found that the peroneus longus tendon (PLT) has sufficient length and strength [11]. Anatomy and biomechanics are similar to PCL, which may have good prospects in reconstructing PCL.

In this prospective study, we tried to analyze the clinical effect of double-stranded long peroneal tendon and the four strands hamstring tendon in reconstruction of PCL, and to investigate the effectiveness and safety of the two surgical approaches in reconstruction of PCL, so as to provide guidance for clinical treatment of PCL injury.

\section{Patients And Methods}

General Information

A total of 48 patients with complete rupture of PCL treated in our hospital from July 2015 to February 2017 were selected and divided into the Group A ( 25 cases) and the Group B ( 23 cases) according to the different grafts. The group A was reconstructed with double strands peroneus longus tendon while and the group B was with four strands hamstring tendon. There were no significant differences between the two groups in sex, age, BMI value or causes of injury (Table I). This study was approved by the Ethics Committee of Affiliated Hospital of Nantong University. Signed written informed consents were obtained from all participants before the study.

PCL diagnosis and exclusion criteria

PCL injury could be classified into three types according to severity: $I^{0}, I^{0}$ and $I I^{0}$. Patients with $I^{0}$ or $I I^{0}$ have complete tendon rupture, which requires surgical treatment. exclusion criteria: (1) Patients with periknee fracture, infection and neurovascular injury; (2) MR imaging confirmed that subjects with other 
stable structural injuries of the knee joint (anterior cruciate ligament (ACL), medial collateral ligament (MCL), etc.). (3) Patients with previous history of knee arthritis or other chronic diseases; patients with severe osteoporosis.

Surgical methods

After routine preoperative preparation and anesthesia, all patients were examined by arthroscopy to determine the injury of patients. In order to obtain the long peroneal tendon, we made a longitudinal incision (proximal incision) about $2 \mathrm{~cm}$ long at the middle and lower $1 / 3$ of fibula in group $A$ to expose the long peroneal tendon. Repair tendon, measure length, fold into two strands after knitting, forming columnar tendon bundle. In group B: The tendon of semitendinosus and gracilis tendon were dissected through a longitudinal incision about $3 \mathrm{~cm}$ long on the medial side of the affected tibial tubercle. The tendon was removed proximally with a closed-mouth key harvester. The tendon was repaired and the length was measured. The semitendinosus and gracilis tendons are woven and folded into four strands ( 2 strands per share, 4 strands in total) to form tendon bundles. The details of the operation and postoperative rehabilitation are described below: The central point of the femoral tunnel is located at 1:30 (right), $12 \mathrm{~mm}$ from the cartilage at the top of the intercondylar fossa, and 7-8 $\mathrm{mm}$ from the nearest cartilage margin at the far side. Tibial tunnel: the outer mouth is located at the medial side of tibial tubercle, and the inner mouth is located at the proximal part of the epiphyseal slope of tibial metaphysis $5 \mathrm{~mm}$. Graft fixation methods was extrusion or suspension of interface screw. When the tendon is installed, the knee joint should be treated at 90 degrees of flexion. After operation, ankle pump exercise, quadriceps isometric contraction exercise, hamstring isometric contraction exercise within 1 week after operation, 2 weeks after the operation, we continued to strengthen the practice of upper operation, passive flexion 60 degrees in case of slight pain, once a day; 3 weeks after the operation, passive flexion 90 degrees, with brace 30 to 50 degrees range of activity, 4 weeks after the operation, passive flexion more than 90 degrees close to 100 degrees, after leg lifting exercise and balance exercise. 5-8 weeks postoperatively, the passive flexion was 110-120 degrees, the static squatting was practiced, 8-10 weeks postoperatively, the passive flexion was 120-130 degrees, 10-12 weeks postoperatively, the gradual passive flexion was the same as that of the healthy side, the sitting position was the same as the resistance knee extension exercise, 3-6 months postoperatively: the active flexion was the same as that of the healthy side, the lower step exercise, the full squatting of the supporting objects, and the physical exercise could be recovered gradually over 7 months postoperatively.

Assessment methods

The analysis of IKDC score [12], Lysholm score [13] and Tegner score [14] was performed in both group A and group B 6 hours before operation and 24 months after operation respectively. KT2000 [15] was also conducted to determine the degree of tibial retroversion preoperative and post-operative (24 months). 
AOFAS score [16] and Maryland score [17] were conducted on the affected limbs 1 day preoperative and 24 months of post-operative to analyze the differences of ankle function.

Statistical analysis

Statistical Product and Service Solutions (SPSS) 17.0 software (SPSS Inc., Chicago, IL, USA) was performed for statistical analysis. The normal distribution data were expressed as (mean $\pm S D)(\bar{x} \pm s)$. Student $t$-test was used for comparison between the two groups, and paired t-test was used for comparison before and after operation.

\section{Results}

\section{Comparison of operational features between the two groups}

The incisions of both groups healed in stage I without complications such as knee joint infection, deep venous thrombosis of lower extremities or neurovascular injury. At 24 months after operation, the PCL healed well on MRI in both groups (Fig. 1-2).

\section{Comparison of intraoperative time for tendon extraction between the two groups}

The intraoperative time for tendon extraction in the Group A was $(20.87 \pm 2.07)$ min, which was shorter than that in the Group B $(26.79 \pm 2.32 \mathrm{~min})$ significantly, as shown in Table II.

\section{The relationship between the IKDC, Lysholm and Tegner score of the two group patients}

The results showed that there was no significant difference in the subjective scores (including the IKDC, Lysholm and Tegner score) between the two group cases before surgery. Comparisons with preoperative statistical results, the IKDC, Lysholm and Tegner scores were significantly higher in both groups 24 months after operation. Nevertheless, there was no difference in IKDC, Lysholm and Tegner scores between the two group subjects (Table III).

\section{Comparison of the KT-2000 between the two group patients}

There was no significant difference in KT-2000, an objective indicator of the knee function, between the both group cases before operation. Comparisons with preoperative statistical results, the posterior tibial displacement was significantly reduced after 24 months of the surgery in both two groups, while no difference was found between the two groups (Table IV). 


\section{Analysis of AOFAS and Maryland score between pre-and post-operation in group A patients}

The AOFAS and Maryland scores, two indicators reflecting ankle joint function between pre- and postoperation, were no significant differences in the group A cases, the results demonstrated that reconstruction with double strands peroneus longus tendon had no side effect on ankle joint function (Table V).

\section{Discussion}

Knee joint, the main load-bearing joint of human lower limbs, plays an important role in transmitting the strength of lower limbs, maintaining flexibility and stability in human daily activities [18]. PCL, as one of the most important ligaments of the knee, has the main function of restricting the posterior tibial displacement and enhancing the stability of the knee joint, as well as limiting tibial rotation, knee joint overextension and adduction (2). Injury of PCL can damage articular cartilage, accelerate joint degeneration, and even lead to osteoarthritis [19]. Therefore, it has become a consensus to reconstruct the stability of knee joint and restore the biological function of knee joint in time after PCL injury.

At present, there are three kinds of reconstruction materials for PCL injury: allogenic tendon, tissue engineered artificial ligament and autogenous tendon. Although allogeneic tendons are widely used in ACL and PCL treatment, they still have defects such as self-rejection, risk of disease transmission and limited contact [20]. LARS artificial ligament has enough strength to resist repeated distortion and excessive traction, and has the advantages of autologous tissue growth. However, because of its high price, it has not been widely used in clinical treatment [21]. The materials for reconstruction of PCL by autogenous tendon transplantation include bone-patellar tendon and hamstring tendon. They all have their own advantages and disadvantages. For example, when using bone-patellar tendon transplantation for reconstruction, the ligament has sufficient strength and stiffness, and can recover quickly after operation. However, patellar fracture, patellar tendon rupture, patellar softening, knee extension weakness and other postoperative complications are prone to occur [22].

The four strands hamstring tendon is a kind of PCL reconstruction graft which is widely used in clinic at present. It has good biological similarity and is favored by clinicians [23]. However, it is located in the functional area of the diseased knee joint. Tendon removal may cause some adverse effects on the stability of the knee joint and potential injury risks such as saphenous nerve injury. The long peroneal tendon is superficial, easy to expose and take out tendons during the operation, and the tendon part is longer. Studies have shown that it is effective in the treatment of tendon injury in other parts [24]. In our study, the time of tendon removal in group $A$ was $20.87 \pm 2.07 \mathrm{~min}$, which was much shorter than that in group $B$, which was $26.79 \pm 2.32$. The results showed that the time of tendon resection in group $A$ was significantly shorter than that in group $B$, and tendon extraction was more convenient and safer. Our results also show that in terms of therapeutic effect, the double-stranded peroneal longus tendon and 
four-stranded hamstring tendon have similar clinical effect in the reconstruction of PCL, which are effective methods for the treatment of cruciate ligament injury.

Several limitations of this study should be noted. First, only 48 patients were selected in this study, that was to say, the sample size was quite small. However, we are going to recruit more patients in our further study. Second, functional performance of the patients determined by gait speed, the timed up and go test (TUTG) test, the sit-to-stand test (STS) and the six-minute walk test (6MWT) have not been carried out in present study. However, we are going to record all these tests in our further study.

\section{Conclusions}

In conclusion, if the tendon condition of some patients cannot meet the requirement of PCL reconstruction in clinical practice, or in PCL patients with other ligament injuries, such as anterior cruciate ligament ( $A C L)$, the single femoral tendon is difficult to meet the surgical requirements. When the hamstring tendon is accidental (tendon rupture or tendon variation) or ineffective, the peroneal longus tendon will be a good choice.

\section{Abbreviations}

ACL: anterior cruciate ligament; MCL: medial collateral ligament; 6MWT: the six-minute walk test; PCL: posterior cruciate ligament; PLT: peroneus longus tendon; STS test: the sit-to-stand test; TUTG test: timed up and go test

\section{Declarations}

\section{Acknowledgements}

Not applicable.

\section{Authors' contributions}

$Y L$ and RT designed the study and performed the experiments, $Y L, H X$ and $Z Z$ collected the data, RT and LF analyzed the data, YL and RT prepared the manuscript. All authors read and approved the final manuscript.

\section{Funding}

Not applicable. 


\section{Availability of data and materials}

The datasets used and analyzed during the current study are available from

the corresponding author on reasonable request.

\section{Ethics approval and consent to participate}

This study was approved by the Ethics Committee of Affiliated Hospital of Nantong University. Signed written informed consents were obtained from all participants before the study.

\section{Consent for publication}

Patients or their guardians have provided written informed consents for publication.

\section{Competing interests}

The authors declare no competing interests.

\section{References}

1. Caldas M, Braga GF, Mendes SL, Da SJ, Kopke RM. Posterior cruciate ligament injury: characteristics and associations of most frequent injuries. Rev Bras Ortop. 2013;48:427-31.

2. LaPrade RF, Pierce CM. Double-Bundle Posterior Cruciate Ligament Reconstruction Technique with Use of Endoscopic Femoral Graft Placement. JBJS Essent Surg Tech. 2012;2:e1.

3. de Souza LM, Santoro ES, Avelino RL, Granjeiro RC, Orlando JN. Combined anterior and posterior cruciate ligaments avulsion from the tibial side in adult patient: case report. Rev Bras Ortop. 2013;48:581-5.

4. Li Y, Hong L, Wang XS, Zhang H, Li X, Zheng T, Feng H. Midterm Clinical Outcome of Combined Posterior Cruciate Ligament Reconstruction and Posterolateral Corner Surgery Using Second-Look Arthroscopic "Lateral Gutter Drive-Through" Test as an Adjunctive Evaluation. Orthop Surg. 2019;11:422-30.

5. Asai K, Nakase J, Oshima T, Shimozaki K, Toyooka K, Tsuchiya H. Lateral meniscus posterior root tear in anterior cruciate ligament injury can be detected using MRI-specific signs in combination but not individually. Knee Surg Sports Traumatol Arthrosc. 2019.

6. Lord BR, El-Daou H, Zdanowicz U, Smigielski R, Amis AA. The Role of Fibers Within the Tibial Attachment of the Anterior Cruciate Ligament in Restraining Tibial Displacement. Arthroscopy. 
2019;35:2101-11.

7. Elnikety S, Pendegrass CJ, de Godoy RF, Holden C, Blunn GW. Augmentation and repair of tendons using demineralised cortical bone. BMC Musculoskelet Disord. 2016;17:483.

8. Wojcik-Pastuszka D, Krzak J, Macikowski B, Berkowski R, Osinski B, Musial W. Evaluation of the Release Kinetics of a Pharmacologically Active Substance from Model Intra-Articular Implants Replacing the Cruciate Ligaments of the Knee. Materials (Basel). 2019; 12.

9. Quan S, Cai L, Yang M. [A modified Laprade technique in treatment of lateral multi-ligament injuries of knee]. Zhongguo Xiu Fu Chong Jian Wai Ke Za Zhi. 2017;31:922-5.

10. Zhao J, He Y, Wang J. Double-bundle anterior cruciate ligament reconstruction: four versus eight strands of hamstring tendon graft. Arthroscopy. 2007;23:766-70.

11. Rhatomy S, Asikin A, Wardani AE, Rukmoyo T, Lumban-Gaol I, Budhiparama NC. Peroneus longus autograft can be recommended as a superior graft to hamstring tendon in single-bundle ACL reconstruction. Knee Surg Sports Traumatol Arthrosc. 2019.

12. Yang XG, Feng JT, He X, Wang F, Hu YC. The effect of knee bracing on the knee function and stability following anterior cruciate ligament reconstruction: A systematic review and meta-analysis of randomized controlled trials. Orthop Traumatol Surg Res. 2019.

13. Devgan A, Rohilla R, Singh A, Tanwar M, Devgan R, Siwach K. A prospective study to evaluate the clinico-radiological outcomes of arthroscopic single bundle versus double bundle anterior cruciate ligament reconstruction. J Clin Orthop Trauma. 2016;7:236-42.

14. Villa FD, Ricci M, Perdisa F, Filardo G, Gamberini J, Caminati D, Villa SD. Anterior cruciate ligament reconstruction and rehabilitation: predictors of functional outcome. Joints. 2015;3:179-85.

15. Huang W, Gong X, Rahul M, Priyanka S, Wang C, Liang X, Ding G, Hu N. Anterior arthroscopic-assisted fixation of posterior cruciate ligament avulsion fractures. Eur J Med Res. 2015;20:88.

16. Gomez-Canedo JM. [Acute rupture of the tibialis anterior tendon]. Acta Ortop Mex. 2017;31:252-6.

17. Bao XX, Xia CJ, Zhao BY, Shi DJ, Lan GH, Ren GL, Xie ZH, Du WX. [Treatment of Sanders II calcaneus fractures via minimally invasive sinus tarsi approach]. Zhongguo Gu Shang. 2017;30:755-8.

18. Ajnin S, Buchanan D, Arbuthnot J, Fernandes R. Patellofemoral joint replacement - Mean five-year follow-up. Knee. 2018;25:1272-7.

19. Hamner DL, Brown CJ, Steiner ME, Hecker AT, Hayes WC. Hamstring tendon grafts for reconstruction of the anterior cruciate ligament: biomechanical evaluation of the use of multiple strands and tensioning techniques. J Bone Joint Surg Am. 1999;81:549-57.

20. Lu W, Xu J, Dong S, Xie G, Yang S, Huangfu X, Li X, Zhang Y, Shen P, Yan Z, Liu H, Deng Z, Zhao J. Anterior Cruciate Ligament Reconstruction in a Rabbit Model Using a Decellularized Allogenic Semitendinous Tendon Combined with Autologous Bone Marrow-Derived Mesenchymal Stem Cells. Stem Cells Transl Med 2019.

21. Iliadis DP, Bourlos DN, Mastrokalos DS, Chronopoulos E, Babis GC. LARS Artificial Ligament Versus ABC Purely Polyester Ligament for Anterior Cruciate Ligament Reconstruction. Orthop J Sports Med. 
2016;4:1807700783.

22. Brown MJ, Carter T. ACL Allograft: Advantages and When to Use. Sports Med Arthrosc Rev. 2018;26:75-8.

23. Tachibana Y, Mae T, Shino K, Ohori T, Amano H, Yoshikawa H, Nakata K. Femoral tunnel enlargement after anatomic anterior cruciate ligament reconstruction: Bone-patellar tendon-bone /single rectangular tunnel versus hamstring tendon / double tunnels. J Orthop Sci. 2018;23:1011-8.

24. Pitts CC, McKissack HM, Anderson MC, Buddemeyer KM, Bassetty C, Naranje SM, Shah A. Anatomical structures at risk in the arthroscopic Brostrom-Gould procedure: A cadaver study. Foot Ankle Surg. 2019.

\section{Tables}

Table I. Comparison of general information between the two groups

\begin{tabular}{lccccccc}
\hline Group & $\mathrm{n}$ & Male & Age (years) & BMI & \multicolumn{3}{c}{ Cause of injury } \\
\cline { 5 - 7 } & & & & Traffic & Sports & Fall \\
\hline Group A & 25 & 11 & $38.23 \pm 10.84$ & $23.76 \pm 2.19$ & 14 & 8 & 3 \\
Group B & 23 & 10 & $36.88 \pm 11.33$ & $23.99 \pm 2.07$ & 13 & 7 & 3 \\
$X^{2} / \mathrm{t}$ & & 0.001 & 0.246 & 0.782 & & 0.020 & \\
$P$ & & 0.975 & 0.597 & 0.211 & & 0.990 & \\
\hline
\end{tabular}

Table II. Comparison of intraoperative time for tendon extraction between the two groups

\begin{tabular}{lcl}
\hline Group & $\mathrm{n}$ & Intraoperative time for tendon extraction (min) \\
\hline Group A & 25 & $20.87 \pm 2.07$ \\
Group B & 23 & $26.79 \pm 2.32$ \\
$t$ & & -30.187 \\
$P$ & & $<0.001$ \\
\hline
\end{tabular}

Table III. Comparison of the IKDC, Lysholm and Tegner score between the two groups 


\begin{tabular}{|c|c|c|c|c|c|}
\hline \multirow[t]{2}{*}{ Group } & \multirow[t]{2}{*}{$\mathrm{n}$} & \multicolumn{2}{|c|}{ IKDC score } & \multirow[t]{2}{*}{$t$} & \multirow[t]{2}{*}{$P$} \\
\hline & & Before operation & After 24 months & & \\
\hline Group A & 25 & $40.37 \pm 4.52$ & $76.46 \pm 4.82$ & -48.215 & $<0.001$ \\
\hline Group B & 23 & $39.23 \pm 4.14$ & $75.12 \pm 4.19$ & -42.124 & $<0.001$ \\
\hline$t$ & & 0.872 & 1.324 & & \\
\hline$P$ & & 0.806 & 0.904 & & \\
\hline \multirow[t]{2}{*}{ Group } & $\mathrm{n}$ & \multicolumn{2}{|c|}{ Lysholm score } & $t$ & $P$ \\
\hline & & Before operation & After 24 months & & \\
\hline Group A & 25 & $52.36 \pm 11.55$ & $86.49 \pm 9.38$ & -18.243 & $<0.001$ \\
\hline Group B & 23 & $53.41 \pm 10.47$ & $85.44 \pm 9.26$ & -24.454 & $<0.001$ \\
\hline$t$ & & 0.092 & 0.055 & & \\
\hline$P$ & & 0.536 & 0.522 & & \\
\hline \multirow[t]{2}{*}{ Group } & $\mathrm{n}$ & \multicolumn{2}{|c|}{ Tegner score } & $t$ & $P$ \\
\hline & & Before operation & After 24 months & & \\
\hline Group A & 25 & $2.78 \pm 0.84$ & $5.34 \pm 0.64$ & -45.342 & $<0.001$ \\
\hline Group B & 23 & $2.52 \pm 0.56$ & $5.42 \pm 0.88$ & -34.232 & $<0.001$ \\
\hline$t$ & & 0.173 & 1.122 & & \\
\hline$P$ & & 0.568 & 0.866 & & \\
\hline
\end{tabular}

Table IV. Comparison of the KT-2000 between the two groups

\begin{tabular}{llllll}
\hline Group & $\mathrm{n}$ & \multicolumn{2}{c}{ KT-2000 (mm) } & $t$ & $P$ \\
\cline { 3 - 4 } & & Before operation & After 24 months & & \\
\hline Group A & 25 & $10.16 \pm 1.85$ & $2.33 \pm 1.32$ & 30.233 & $<0.001$ \\
Group B & 23 & $10.92 \pm 1.93$ & $2.56 \pm 1.53$ & 37.238 & $<0.001$ \\
$t$ & & 1.815 & 0.988 & & \\
$P$ & & 0.962 & 0.836 & & \\
\hline
\end{tabular}

Table V. Comparison of AOFAS and Maryland score between pre- and post-operation in group A

\begin{tabular}{lll}
\hline Group A & AOFAS score & Maryland score \\
\hline Before operation & $87.36 \pm 2.12$ & $89.40 \pm 5.87$ \\
After 24 months & $86.00 \pm 3.19$ & $88.99 \pm 4.09$ \\
$t$ & 0.623 & 1.288 \\
$P$ & 0.732 & 0.898 \\
\hline
\end{tabular}


Figures
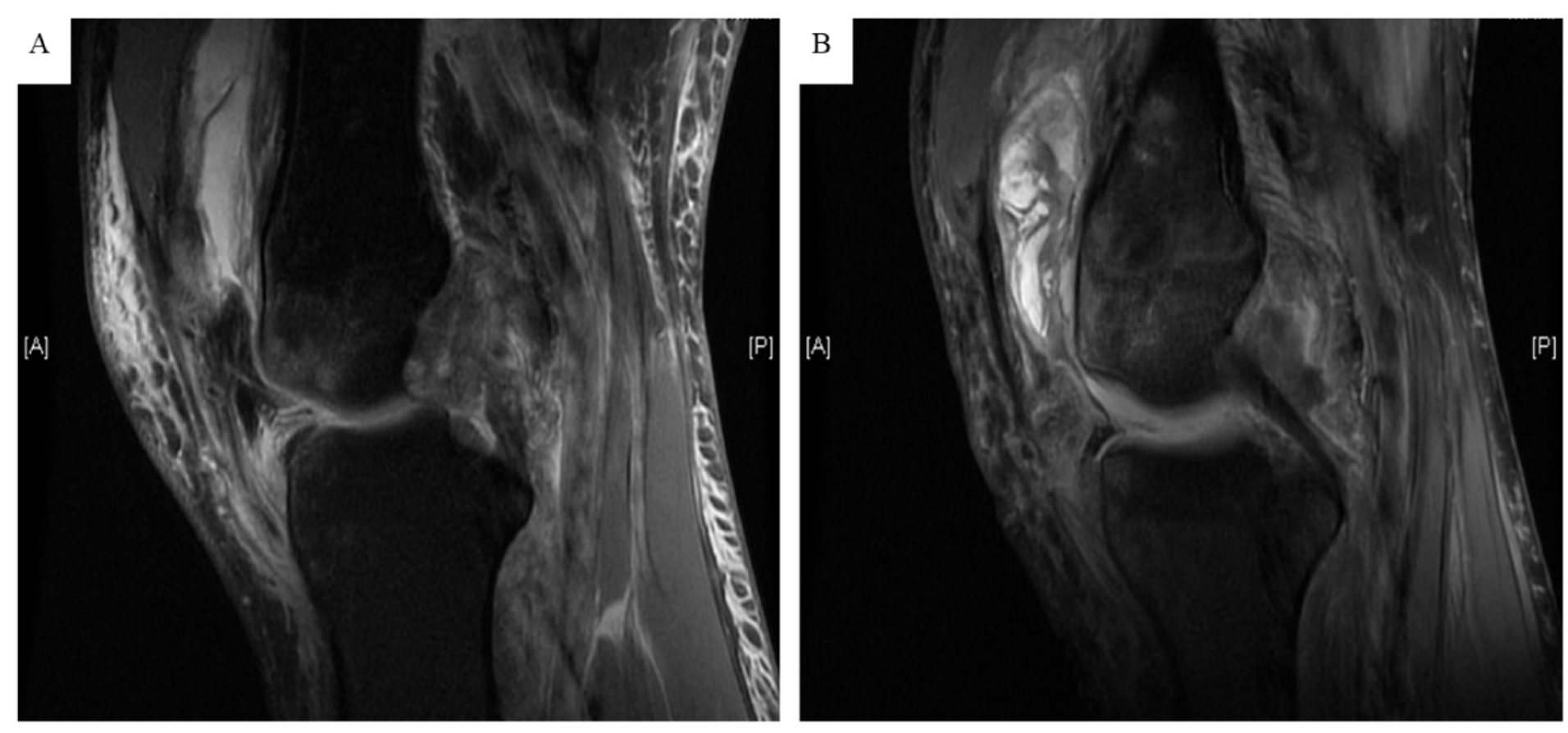

\section{Figure 1}

The MRI of PCL injury preopertaion and 24 months after operation by reconstructed with double strands peroneus longus tendon.
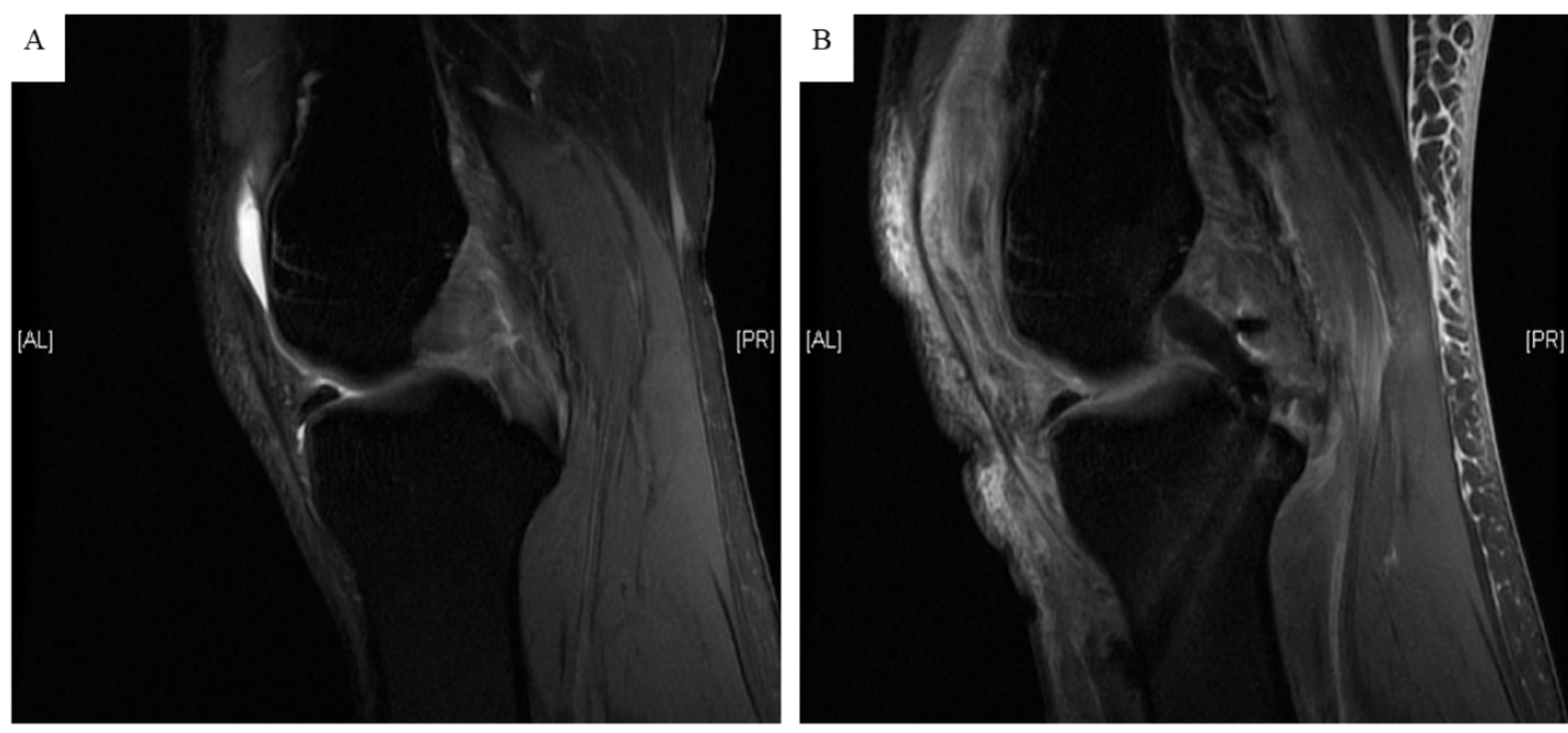

Figure 2 
The MRI of PCL injury preopertaion and 24 months after operation by reconstructed with four strands hamstring tendon. 